\title{
Laser printed flat lenses for optofluidics
}

\author{
Airidas Žukauskas ${ }^{1, *}$, Andreas R. Stilling-Andersen ${ }^{1}$, Xiaolong $\mathrm{Zhu}^{1}$, and Anders Kristensen ${ }^{1}$ \\ ${ }^{1}$ DTU Health Tech, Technical University of Denmark, DK-2800 Kongens Lyngby, Denmark
}

\begin{abstract}
Conventional three-dimensional optics requires curvature to control the wave front of light thus making it difficult to reduce the size of the optical systems. Furthermore, for correction of optical aberrations, complex optical systems comprising more than one lens are used. This adds additional bulk, mass and complexity to the optical systems. Recent development in diffractive optics has enabled new thin lightweight optical elements such as metalenses. We introduce resonant laser printing technique as a flexible photo-thermal technology for metalens fabrication with the ability to control the light with microscale precision. Our laser printed metalenses can be integrated in bio-sensors, bio-imaging systems, and optofluidical devices.
\end{abstract}

\section{Introduction}

Optical metasurfaces are arrays of sub-wavelength sized elements - meta-atoms - enabling precise control of phase, amplitude and polarization of light [1]. These metasurfaces are made from metallic (e.g. aluminum [2]) or highindex (e.g. silicon [3]) nano or micro sized arrays of metaatoms that gives the surface unique optical features. These properties are defined by the material composition, the geometry and the orientation. These arrays are usually fabricated by Electron-beam [4] or Deep UltraViolet [5] lithography techniques which are multi-step, time consuming and nonflexible, making the flats lenses expensive and difficult to fabricate.

We introduce resonant laser printing (RLP) as a technique for fast and precise metasurface modification [6-9]. Nanosecond laser pulses are used for this process as the meta-atoms absorb the laser pulses and converts the energy to heat, which raises the temperature of the meta-atoms and causes them to melt and solidify in a short time period. This process changes the geometry of the structure and results in new optical properties of the metasurface. By controlling the laser pulse energy, it is possible to precisely tune the dewetting process.

We created metasurfaces with double resonances: one resonance in the visible - at the wavelength of the laser, we used for writing - to improve laser writing efficiency by decreasing the laser power required to modify the metaatoms. The other resonance is the transmission resonance at the infra-red operating wavelength - that is very important for our flat lenses to work. Laser induced photothermal melting of the meta-atoms shifts the resonance at the infra-red operation wavelength, when the geometry of the meta-atom changes, it causes the resonance in transmission to blue shift, thus making the area more transparent.
Combining pre-fabricated, large area metasurface templates and the resonant laser printing technique, we get a low-cost and flexible fabrication technique of the metalenses that can be used for biological sensing, biological imaging, and optical trapping.

\section{Results and discussion}

In this work, we used pre-fabricated silicon based metasurface templates with double resonances at $532 \mathrm{~nm}$ and at $1064 \mathrm{~nm}$. These templates were used to resonant laser print binary amplitude based Fresnel zone plates. We designed our metalens to work at a wavelength of 1064 $\mathrm{nm}$ (Fig. 1) with the focus length: $100 \mu \mathrm{m}$, number of rings/zones: 32, numerical aperture (NA): 0.7.

The laser written metalens was characterized by a custom built setup (Fig. 2), and we measured a focus length of $113 \mu \mathrm{m}$, beam FWHM of $2.29 \mu \mathrm{m}$ at focus, the efficiency of the lens: $16.98 \%$, and the lens diameter was $122 \mu \mathrm{m}$. Because of its small size, it can be integrated in optofluidics devices [10].
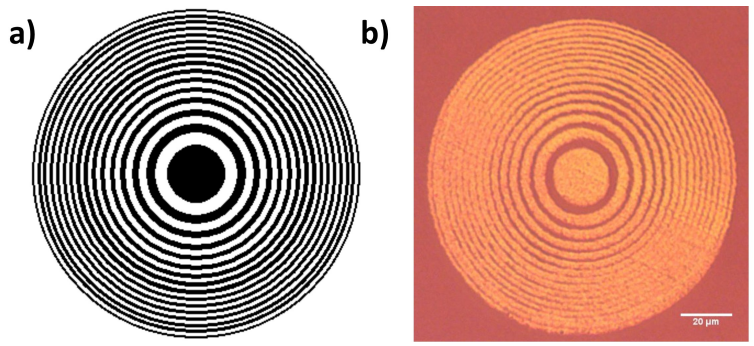

Figure 1. a) Our metalens design with the focus length: $100 \mu \mathrm{m}$, ring number: 32 , numerical aperture (NA): 0.7, $305 \times 305$ pixels image. b) Transmission optical microscope image of the laser written metalens onto silicon based metalens templates. The scale bar is $20 \mu \mathrm{m}$.

*e-mail: aiuk@dtu.dk 




Figure 2. Schematics of out metalens characterization setup. 1) $1064 \mathrm{~nm}$ diode laser, 2) Glan-Thompson polarizer, 3) $\lambda / 4$ plate, 4) motorized $X Y Z$ and rotation stage with sample holder, 5) sample, 6) 20× objective, 7) $f=150 \mathrm{~mm}$ lens, 8) CCD camera, 9) $\mathrm{XYZ}$ stage.

\section{Conclusions}

In conclusion, we demonstrated a powerful resonant laser writing technique for ultra-thin micro sized metalenses fabrication. These metalenses can be used for bio-imaging or for bio-sensors. Because of the very small size, it is possible to integrate these metalenses into optofluidic devices. Using prefabricated templates and laser fabrication, the cost of metalens is low, which provides a path for massproduction and ready-for-applications processes.

\section{Acknowledgements}

This work was supported by the European Commission project CHROMAVISION (Grant Agreement No.
665233). X.Z. is funded by VILLUM Experiment (grant No. 17400) supported by VILLUM FONDEN.

\section{References}

[1] N. Yu, F. Capasso, Nature Materials 13, 139 (2014)

[2] E. Hjlund-Nielsen, X. Zhu, M.S. Carstensen, M.K. Sørensen, C. Vannahme, N.A. Mortensen, A. Kristensen, Optics Express 23, 28829 (2015)

[3] Z. Zhou, J. Li, R. Su, B. Yao, H. Fang, K. Li, L. Zhou, J. Liu, D. Stellinga, C.P. Reardon et al., Acs Photonics 4, 544 (2017)

[4] F. Yue, D. Wen, J. Xin, B. Gerardot, J. Li, X. Chen, p. 17 (2016)

[5] M. Iwanaga, 2016 Progress in Electromagnetics Research Symposium, Piers 2016 - Proceedings pp. 7734815, 1857-1861 (2016)

[6] X. Zhu, C. Vannahme, E. Højlund-Nielsen, N.A. Mortensen, A. Kristensen, Nature Nanotechnology 11, 325 (2016)

[7] X. Zhu, W. Yan, U. Levy, N.A. Mortensen, A. Kristensen, Science Advances 3, e1602487, e1602487 (2017)

[8] X. Zhu, M. Keshavarz Hedayati, S. Raza, U. Levy, N.A. Mortensen, A. Kristensen, Nano Today 19, 7 (2018)

[9] M.S. Carstensen, X. Zhu, O.E. Iyore, N.A. Mortensen, U. Levy, A. Kristensen, A C S Photonics 5, 1665 (2018)

[10] A. Žukauskas, X. Zhu, A. Kristensen (2019), in progress 